\title{
LUNA-400 and LUNA-MV: present and future of Nuclear Astrophysics at LNGS
}

\author{
Carlo Gustavino* \\ INFN-ROMA \\ E-mail: carlo.gustavinoeromal.infn.it
}

\begin{abstract}
The understanding of our universe depends on the knowledge of the cross section of many nuclear reactions at low energy. In fact the existence of all the celestial bodies and their evolution is regulated by gravitation and specific thermonuclear reaction rates. Similarly, the light isotopes produced during the big bang nucleosynthesis are the result of the competition between the expanding universe and the cross sections of relevant nuclear processes. At energy of interest in nuclear astrophysics the cross sections are very low because of the Coulomb repulsion between the interacting nuclei. For this reason, nuclear astrophysics measurements are severely hampered by the radioactivity induced by cosmic rays. The natural solution is to study the reaction of astrophysical interest deep underground, were the background can be reduced by orders of magnitude with respect to facilities operating at the earth surface. LUNA (Laboratory for Underground Nuclear Astrophysics) is the worldwide only facility for nuclear astrophysics operating deep underground. This paper reports outstanding results obtained by LUNA and their implication in astrophysics, particle physics and cosmology. The future developments of the LUNA experiment are also discussed.
\end{abstract}

53rd International Winter Meeting on Nuclear Physics,

26-30 January 2015

Bormio, Italy

\footnotetext{
*for the LUNA colaboration
} 


\section{Introduction}

The synthesis of elements in stars are due to thermonuclear fusion reactions, which start from the most abundant and lightest element, the hydrogen, towards to the synthesis of the heaviest elements. Therefore nuclear fusion reactions determine the energy production, the neutrino luminosity and the evolution of stars. In the stellar environment the thermal energy of nuclei is much lower than the Coulomb barrier. As an example, the core temperature of the Sun is about $15 \cdot 10^{6} \mathrm{~K}$, corresponding to a Maxwell-Boltzmann energy distribution peaked to about $1 \mathrm{keV}$, much lower than the $550 \mathrm{keV}$ effective Coulomb barrier for the $p+p$ reaction. Indeed, the nuclear reactions take place via quantum tunneling effect and the cross section of charged particle reactions is often factorized as follows:

$$
\sigma(E)=\frac{S(E) e^{-2 \pi \eta}}{E}
$$

In this formula, the exponential term takes into account the Coulomb barrier, while the astrophysical factor $S(E)$ contains all the nuclear effects. For non-resonant reactions, it is a smoothly varying function of energy. The Sommerfeld parameter $\eta$ is given by $2 \pi \eta=31.29 Z_{1} Z_{2}(\mu / E)^{1 / 2} \cdot Z_{1}$ and $Z_{2}$ are the nuclear charges of the interacting nuclei. $\mu$ is their reduced mass (in units of a.m.u.), and $E$ is the center of mass energy (in units of $\mathrm{keV}$ ) [1,2]. The fusion reactions in stars occur in a well defined energy range, the so called Gamow peak, which arises from the convolution of the thermal energy distribution of nuclei and the tunneling probability through the Coulomb barrier between the interacting nuclei. For a given stellar temperature $T$ the energy corresponding to the Gamow peak is larger than $k T$, typically in the order of tens of $k e V$. Most of the reactions between light nuclei at such energies have cross sections of the order of $10^{-9} \div 10^{-12}$ barn. These correspond, under typical laboratory conditions, to experimental counting rates ranging from a few events per day to a few events per month. This means that in realistic experimental conditions, the expected counting rate is prohibitively low with respect to the detector background induced by the cosmic rays. A drastic reduction of this background can be achieved by carrying out the measurement deep underground. The "Laboratori Nazionali del Gran Sasso" (LNGS) hosts the facility LUNA (Laboratory for Underground Nuclear Astrophysics), that is the worldwide unique facility installed deep underground, in which cross sections at energies of astrophysical interest are measured. The average depth of $1400 \mathrm{~m}$ of rock leads to a reduction of the muon and neutron fluxes by a factor $10^{6}$ and $10^{3}$, respectively. The suppression of the cosmic ray induced background also allows an effective suppression of the $\gamma$-ray activity by a factor $10^{3} \div 10^{5}$, depending on the photon energy.

\section{The accelerators}

High beam current, long-term stability, and precise beam-energy determination are the requirements for an ion accelerator conceived for direct nuclear astrophysics measurements. The first feature maximizes the reaction rate, the second provides the long time typically needed for a cross-section measurement, and the third is important because of the exponential energy dependency of the cross section (see eq. 1.1). The very first accelerator used at LUNA was a $50 \mathrm{kV}$ machine, consisting of a duoplasmatron ion source, an ion-beam extraction and acceleration system, and a double-focusing $90^{\circ}$ analyzing magnet. This machine was designed to deliver beams of protons, ${ }^{3} \mathrm{He}$ and ${ }^{4} \mathrm{He}$ ions of about $400 \mu \mathrm{A}$ at energies between 10 and $50 \mathrm{keV}$ with an energy 


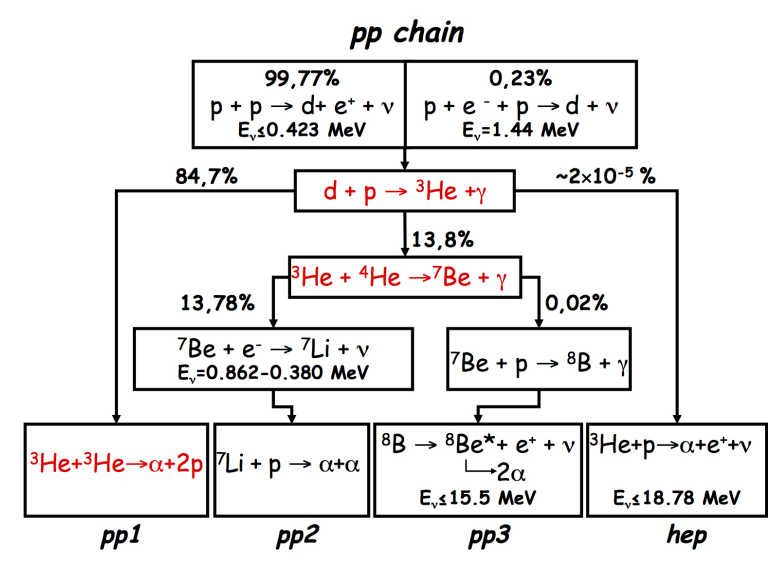

Figure 1: Scheme of the proton-proton reaction chain. The processes studied by the LUNA collaboration are in red.

spread of less than $20 \mathrm{eV}$ and a long-term stability [3]. With this accelerator have been measured, for the first time inside the solar Gamow peak, the cross section of two fundamental reactions of the proton-proton (p-p) chain, namely the ${ }^{3} \mathrm{He}\left({ }^{3} \mathrm{He}, 2 p\right){ }^{4} \mathrm{He}$ and ${ }^{2} \mathrm{H}(p, \gamma){ }^{3} \mathrm{He}$ reactions [4, 5]. This accelerator was also used to study the screening effect in the ${ }^{2} \mathrm{H}\left({ }^{3} \mathrm{He}, p\right){ }^{4} \mathrm{He}$ reaction $[6,7,8]$. Even though the LUNA-50 accelerator has produced outstanding results, it should be considered as a pilot project toward the present facility, the LUNA-400 accelerator [9]. The high voltage is generated by an inline Cockcroft-Walton power supply. The radio frequency ion source directly mounted on the accelerator tube can provide $500 \mu \mathrm{A}$ beams of protons or $\alpha$ particles up to $400 \mathrm{keV}$, over a continuous operating time of 40 days, yielding $0.3 \mathrm{keV}$ accuracy on the beam energy, $100 \mathrm{eV}$ energy spread. The ions can be sent into one of two different, parallel beam lines, thereby allowing the installation of two different target setups. The first beam line is equipped with solid targets. A solid target may contain a large number of atoms per square centimeter: Typical areal densities range from $2 \times 10^{17}$ to $2 \times 10^{18}$. They allow for the measurement of the cross section angular dependency because the beam-hit position on the target can be precisely determined. Solid targets, in turn, may deteriorate under intense beams. The second beam line is equipped with a windowless gas target (i.e., a differentially pumped target) and a recirculation system. Gas targets are more stable to beam bombardment and may reach extreme purity, which is essential to minimize the beam-induced background. However, the areal densities used in LUNA are somewhat smaller with respect solid targets, between $5 \times 10^{16}$ to $1 \times 10^{18}$. As it is summarized in table 1 , the LUNA-400 accelerator is mainly employed to study reactions of hydrogen burning in the Sun and in other celestial bodies, e.g. massive stars, AGB stars, Novae.

Although the LUNA-400 accelerator is presently full operative, the future of LUNA is addressed to study the successive phases of stars, towards the synthesis of heavier and heavier elements. This program can be accomplished with an accelerator operating in the several $\mathrm{MeV}$ region. In this concern The LUNA-MV project has been approved and funded, and will be operative in few years. This machine is conceived to deliver beams of protons, ${ }^{4} \mathrm{He}$ and ${ }^{12} \mathrm{C}$ up to $4.5 \mathrm{MeV}$. The starting program foresees the study of the ${ }^{12} C(\alpha, \gamma){ }^{16} O$ reaction, that is of a primary importance for the helium burning phase of the stars. The ${ }^{13} \mathrm{C}(\alpha, n){ }^{16} \mathrm{O}$ and the ${ }^{22} \mathrm{Ne}(\alpha, n){ }^{25} \mathrm{Mg}$ reactions will be 
Table 1: Selection of nuclear reaction already studied by LUNA (a), in progress (b) and in program (c).

\begin{tabular}{|c|c|c|c|}
\hline Reaction & main motivation & accelerator & reference \\
\hline${ }^{3} \mathrm{He}\left({ }^{3} \mathrm{He}, 2 p\right)^{4} \mathrm{He}^{a}$ & p-p chain & LUNA-50 & [4] \\
\hline$d\left({ }^{3} \mathrm{He}, p\right)^{4} \mathrm{He} e^{a}$ & electron screening, BBN & LUNA-50 & {$[6,7,8]$} \\
\hline$d(p, \gamma)^{3} H e^{a, b}$ & $\mathrm{BBN}$, proto-stars & LUNA-50, LUNA-400 & {$[5,32,29]$} \\
\hline${ }^{3} \mathrm{He}(\alpha, \gamma)^{7} \mathrm{Be} e^{a}$ & p-p chain, BBN & LUNA-400 & {$[12,13]$} \\
\hline${ }^{14} N(p, \gamma){ }^{15} O^{a}$ & CNO cycle I & LUNA-400 & {$[14,15,16,17,18]$} \\
\hline${ }^{25} M g(p, \gamma)^{26} A l^{a}$ & Mg-Al cycle & LUNA-400 & {$[19,20,21]$} \\
\hline${ }^{15} N(p, \gamma){ }^{16} O^{a}$ & CNO cycle II & LUNA-400 & {$[22,23]$} \\
\hline${ }^{17} O(p, \gamma)^{18} F^{a}$ & CNO cycle III & LUNA-400 & {$[24,25]$} \\
\hline${ }^{22} \mathrm{Ne}(p, \gamma){ }^{23} \mathrm{Na}^{b}$ & $\mathrm{Ne}-\mathrm{Na}$ cycle & LUNA-400 & {$[26]$} \\
\hline${ }^{18} O(p, \gamma){ }^{19} F^{b}$ & CNO cycle IV & LUNA-400 & {$[26]$} \\
\hline${ }^{23} \mathrm{Na}(p, \gamma)^{24} \mathrm{Mg}{ }^{b}$ & $\mathrm{Ne}-\mathrm{Na}$ cycle & LUNA-400 & [26] \\
\hline$d(\alpha, \gamma){ }^{6} L i^{a, c}$ & BBN & LUNA-400, LUNA-MV & {$[27,28]$} \\
\hline${ }^{13} C(\alpha, n){ }^{16} O^{c}$ & S-process & LUNA-400, LUNA-MV & {$[29,30,31]$} \\
\hline${ }^{22} \mathrm{Ne}(\alpha, n)^{25} \mathrm{Mg}^{c}$ & S-process & LUNA-MV & {$[30,31]$} \\
\hline${ }^{12} C(\alpha, \gamma){ }^{16} O^{c}$ & Helium burning & LUNA-MV & {$[30,31]$} \\
\hline${ }^{12} C+{ }^{12} C$ reactions ${ }^{c}$ & Carbon burning & LUNA-MV & \\
\hline
\end{tabular}

also studied, to define the neutron production in stars and the inset of S-process. Finally the study ${ }^{12} \mathrm{C}+{ }^{12} \mathrm{C}$ processes are also of high interest, to explore the Carbon burning stage of the stars (see also tab. 1).

\section{The Hydrogen burning in the Sun}

The energy produced in the Sun is the product of nuclear reactions in which four protons are transformed into an $\alpha$ particle, 2 positrons and 2 neutrinos. In the Sun this synthesis is predominantly due to the proton-proton chain (p-p chain), while the contribution of the CNO cycle is at the level of $1 \%$ [10]. Figure 1 shows the p-p chain. About $86 \%$ of neutrinos are produced by the $p\left(p, e^{+} v\right) D$ reaction (p-p neutrinos, $E_{v} \leqslant 0.423 \mathrm{MeV}$ ). The isotope ${ }^{4} \mathrm{He}$ is mainly produced by the ${ }^{3} \mathrm{He}\left({ }^{3} \mathrm{He}, 2 p\right){ }^{4} \mathrm{He}$ reaction (pp1 chain, see figure 1). However, a relevant fraction of ${ }^{4} \mathrm{He}$ is generated through the ${ }^{3} \mathrm{He}(\alpha, \gamma){ }^{7} \mathrm{Be}$ reaction. The ${ }^{7} \mathrm{Be}$ can go down two different paths from here. In the pp2 chain the berillium captures an electron producing mono energetic neutrinos ( $E_{V}=0.370$ and $E_{V}=0.862 \mathrm{MeV}$, respectively with a Branching ratio of $10 \%$ and $90 \%$ ). This reaction produces $14 \%$ of the solar neutrinos. In the pp 3 chain the ${ }^{7} B e$ reacts with a proton producing ${ }^{8} B$. The ${ }^{8} B$ decays into ${ }^{8} \mathrm{Be}$ producing high energy neutrinos $\left(E_{v} \leqslant 15.5 \mathrm{MeV}\right)$. This reaction produces about $0.02 \%$ of the solar neutrinos. The subsequent decay of ${ }^{8} B e$ produces two $\alpha$ particles. The CNO cycle (see figure 2) contribute to the hydrogen burning in the Sun and its neutrino luminosity in a much lower extent (about 1\%).

In conclusion, the total solar energy production and neutrino luminosity is essentially determined by the p-p chain, whose rate is regulated by the weak processes that convert two protons into deuterium. The ratio of low energy neutrinos flux (pp neutrinos, see figure 3) and the high energy beryl- 


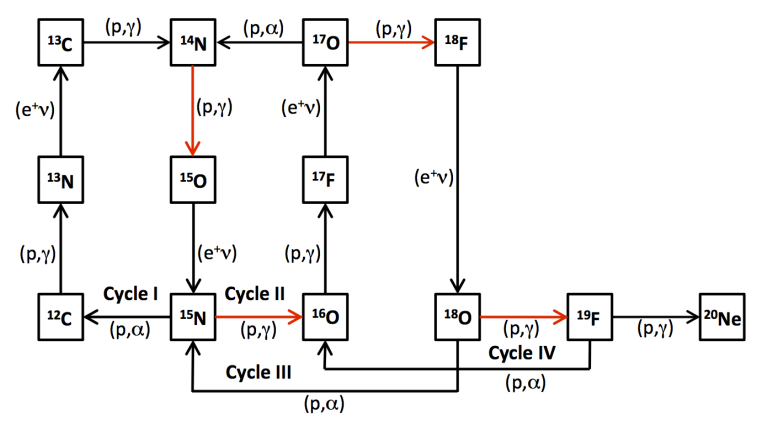

Figure 2: Scheme of the CNO cycles. The red arrows indicate the reactions studied by LUNA.

lium and boron neutrino fluxes depends on the reaction rate ratio between the ${ }^{3} \mathrm{He}\left({ }^{3} \mathrm{He}, 2 p\right){ }^{4} \mathrm{He}$ and ${ }^{3} \mathrm{He}(\alpha, \gamma)^{7} \mathrm{Be}$ processes. The ratio between the boron and beryllium neutrino fluxes depends on the reaction rate ratio between the ${ }^{7} B e\left(e^{-}, v\right){ }^{7} L i$ and ${ }^{7} B e(p, \gamma){ }^{8} B$ processes. Finally, the neutrino flux due to the CNO cycle depends on the metallicity of the SUN (i.e. the abundance of heavy elements such as carbon, nitrogen and oxygen) and on the ${ }^{14} N(p, \gamma){ }^{15} \mathrm{O}$ reaction rate, because it is the slowest process in the CNO cycle.

The first observation of solar neutrinos due to the Davis experiment, in which the detection of $v_{e}$ neutrinos was performed with a Chlorine target. The experimental result was a deficit of about a factor 3 with respect to the Standard Solar Model (SSM) prediction. Afterwards a neutrino deficit was confirmed by the water-Cerenkov Kamioka experiment and by the Gallium experiments (Gallex and Sage). A conservative way to explain the solar neutrino deficit (before the neutrino oscillation discovery) was the theoretical possibility of the existence of a narrow resonance inside the solar Gamow peak of the ${ }^{3} \mathrm{He}\left({ }^{3} \mathrm{He}, 2 p\right){ }^{4} \mathrm{He}$ reaction [11]. As explained above, such a resonance enhances the rate of low energy neutrinos, at the expense of high energy neutrinos produced in the pp2 and pp3 chains. In such a way it was possible to explain the neutrino deficit observed by the solar neutrino experiments, mainly sensitive to the high energy neutrinos flux (see figure 3). In this framework, the achievement of measuring the the ${ }^{3} \mathrm{He}\left({ }^{3} \mathrm{He}, 2 \mathrm{p}\right)^{4} \mathrm{He}$ cross section inside the solar Gamow peak can be considered a milestone in the long way to the discovery of neutrino oscillation. The existence of the Fowler resonance to explain the "Solar neutrino problem" was definitively rejected, pushing towards a new generation of experiments addressed to measure the neutrino oscillation parameters and the solar interior (Borexino, Kamland, SNO). Figure 4 shows the data of the astrophysical factor $S(E)$ performed by LUNA. It is worth to point out that at energy of $E_{c m}=16.5 \mathrm{keV}$ the measured cross section is $0.02 \pm 0.02 \mathrm{pb}$, which corresponds to a rate of about 1 event/month, rather low even for the "silent" experiments of underground physics [4].

After the discovery of the solar neutrino oscillation, the main goal of the solar neutrino experiments is presently the study of the solar interior. As an example, the Boron neutrino flux is an excellent "thermometer" of the core of the Sun, in fact it is proportional to $T_{\odot}^{20}$, where $T_{\odot}$ is the temperature of the core of the Sun. As stated above, the ${ }^{8} B$ neutrino flux depends on the accurate knowledge of the reactions ${ }^{3} \mathrm{He}\left({ }^{4} \mathrm{He}, \gamma\right){ }^{7} \mathrm{Be}$. Before the LUNA measurement, the main source of uncertainty was the difference between direct measurements, where prompt $\gamma$ rays are detected, and indirect measurements, where the $\gamma$ 's yield from the decay of ${ }^{7} \mathrm{Be}$ (half-life of $53.22 \pm 0.06$ days) 


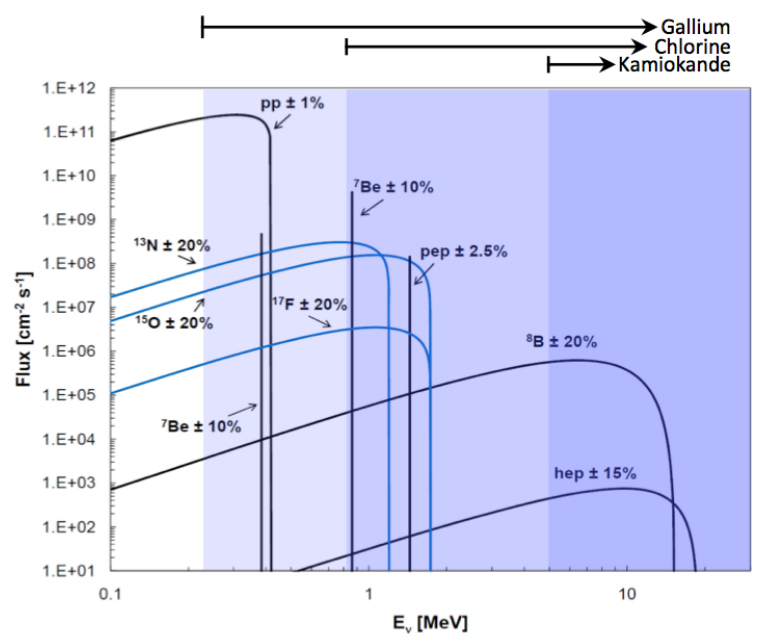

Figure 3: neutrino flux Vs energy in the Sun. The thresholds of Gallium, Chlorine and Kamiokande experiments are also shown.

is measured. Figure 5 shows the astrophysical factor of this reaction measured by LUNA and other experiments. The study of LUNA provides data at the lowest energies and with the best accuracy at the same time, by detecting both the yields of prompt and delayed $\gamma$ 's. With this measurement, the uncertainty of the ${ }^{8} B\left({ }^{7} \mathrm{Be}\right)$ calculated neutrino flux due to $S_{34}$ has been reduced from $7.5 \%(8.0 \%)$ to $2.4 \%(2.5 \%)$. The overall uncertainty, including the astrophysical parameters, goes from $12 \%$ $(9.4 \%)$ to $10.0 \%(5.5 \%)$ [13].

Although the CNO cycle is less effective for the hydrogen burning is the Sun, the neutrinos produced in this way are very important because they are a direct probe to measure the solar metallicity [10]. The measurement of the CNO neutrino flux is therefore one of the main goal of the Borexino experiment, to establish the abundance of "metals" in the Sun. As stated above, the rate of the CNO cycle depends almost linearly by the slowest process of the cycle, the ${ }^{14} N(p, \gamma){ }^{15} O$ reaction. This reaction has been measured by LUNA in several steps and with different techniques, with the aim of reaching an energy close to the Gamow peak region. The partial cross sections involving the complex ${ }^{15} \mathrm{O}$ level structure, have also been measured at low energy, to obtain a reliable extrapolation of the astrophysical S-factor [14, 17]. The lowest energy reached by LUNA was $70 \mathrm{keV}$, which corresponds to about 11 counts/day, with a cross section of about $0.2 p b$ [18]. As shown in figure 6, the LUNA measurement establishes that the astrophysical factor, extrapolated at zero energy, is a factor 2 lower with respect to previous estimation. The direct consequence for the SSM is the halving of estimated CNO neutrino flux with respect to previous estimations.

An important consequence of the LUNA cross section measurement of ${ }^{14} N(p, \gamma){ }^{15} O$ reaction is the revision of the globular cluster age. Globular clusters (GC) are considered the oldest resolved stellar populations. For this reason the stars observed in GC are ancient first generation stars, with masses smaller than that of the Sun. Therefore, GC are used to give an estimation of the age of universe independent from the one obtained by measuring the rate of expansion of the universe and extrapolating back to the Big Bang (PLANCK collaboration quote $t_{0}=13.82 \pm 0.06 \mathrm{Gyr}$ ). As it is 


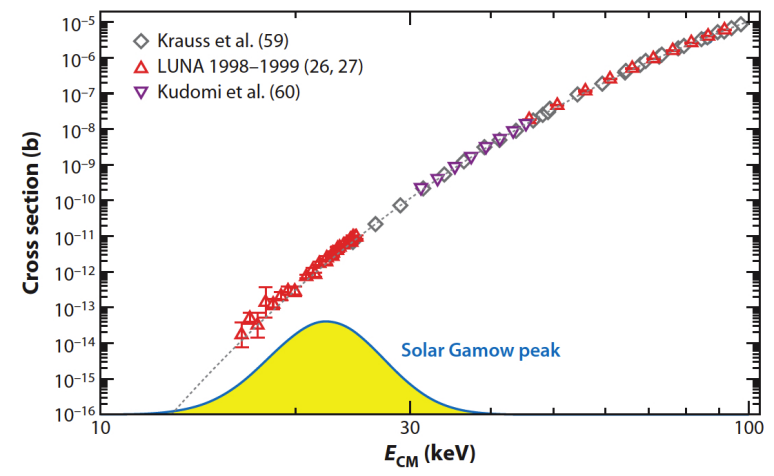

Figure 4: Cross section of the ${ }^{3} \mathrm{He}\left({ }^{3} \mathrm{He}, 2 p\right){ }^{4} \mathrm{He}$ reaction. The LUNA measurements (filled and empty circles) are shown together with previous measurements (see [4] and references therein). The solar Gamow peak region is also shown.

the case of the Sun, these low mass stars burn hydrogen in the center mainly through the p-p chain. Towards the end of their life, when the mass fraction of hydrogen in the core becomes smaller than about $10 \%$, the stellar core contracts, the central temperature increases and the hydrogen burning switches from the p-p chain to the more efficient $\mathrm{CNO}$ cycle. Thus, the departure from the main sequence is powered by the CNO cycle, whose bottleneck is the ${ }^{14} N(p, \gamma){ }^{15} O$ reaction. The luminosity of the turnoff depends on the astrophysical S-factor of this key reaction: the larger the rate, the fainter the turnoff. The revised astrophysical factor due to the LUNA data results in an increase of the GC age of about $0.7 \div 1 \mathrm{Gyr}$ with respect to the previous estimates. With the present LUNA results, the age of universe inferred from the GC age is around $14.5 \mathrm{Gyr}$, with an uncertainty of about of 1 Gyr [15].

\section{Other hydrogen burning cycles}

In stars somewhat more massive than the Sun, the hydrostatic equilibrium is reached at higher densities and temperatures, in such a way the hydrogen burning take place also through cycles involving heavier elements with respect to the CNO I-II cycles, such as the CNO III-IV cycles (see figure 2), the $\mathrm{Ne}-\mathrm{Na}$ and the $\mathrm{Mg}$-Al cycles. Because of the higher Coulomb barriers involved in these reactions, these cycles are relatively unimportant as additional energy sources in stars, but are important, however, for the nucleosynthesis of many elements and isotopes and therefore to understand the chemical composition of Universe. As shown in table 1, the LUNA program foresees the study of several key reaction of hydrogen burning in hot environments, namely the ${ }^{17} \mathrm{O}(p, \gamma){ }^{18} \mathrm{~F},{ }^{18} \mathrm{O}(p, \gamma){ }^{19} \mathrm{~F},{ }^{23} \mathrm{Na}(p, \gamma){ }^{24} \mathrm{Mg}$ and ${ }^{25} \mathrm{Mg}(p, \gamma){ }^{26} \mathrm{Al}$ reactions.

\section{Advanced burning}

The evolution and fate of a star basically depends on its mass and its initial chemical composition. The hydrogen burning generate a helium-rich core that contracts slowly, until its density and temperature reach the level to inset the helium burning stage. Helium burning begins forming ${ }^{12} C$ through the triple- $\alpha$ process. In turn, the ${ }^{12} C$ can capture another $\alpha$ particle through the 


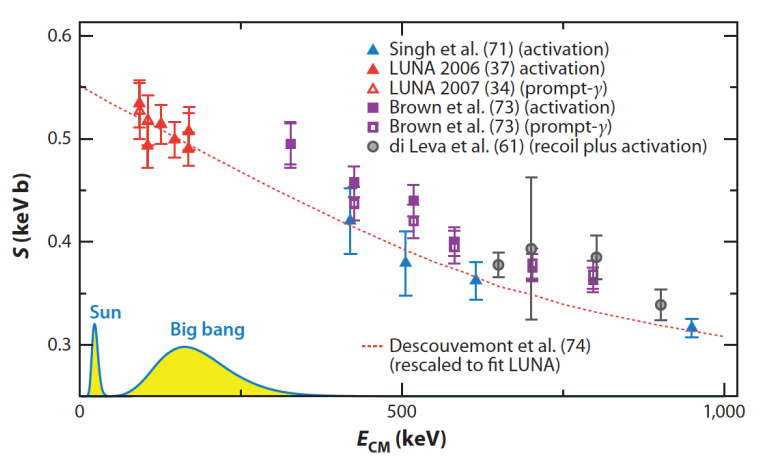

Figure 5: Astrophysical factor of the ${ }^{3} \mathrm{He}\left({ }^{4} \mathrm{He}, \gamma\right){ }^{7} \mathrm{Be}$. The LUNA measurements (filled and empty circles) are shown together with previous measurements (see [13] and references therein).

${ }^{12} C(\alpha, \gamma){ }^{16} O$ process. The ${ }^{12} C(\alpha, \gamma){ }^{16} O$ determines the production of carbon and oxygen, elements of primary importance in biological processes. The helium burning causes the development of a core composed primarily by carbon and oxygen. For sufficiently massive stars, the gravitational collapse causes the further increase of density and temperature to ignite the carbon. indeed, through the ${ }^{12} \mathrm{C}+{ }^{12} \mathrm{C}$ reactions, is produced neon, sodium and magnesium. Heavier elements are produced in the successive stage of stellar evolution, until the explosive burning. However, also stars with small and intermediate mass, which evolve into white dwarfs, may originate under particular conditions violent phenomena powered by thermonuclear explosions, such as novae and supernovae of type Ia. The detailed evolution of such phenomena also depends on the C/O ratio and therefore by the ${ }^{12} \mathrm{C}(\alpha, \gamma){ }^{16} \mathrm{O}$ cross section [1,2]. Finally, two reactions are of particular importance during the advanced burning, the ${ }^{13} \mathrm{C}(\alpha, n){ }^{16} \mathrm{O}$ and the ${ }^{22} \mathrm{Ne}(\alpha, n){ }^{25} \mathrm{Mg}$ reactions. In fact their are responsible for the neutron production and therefore inset the "slow neutron capture process" (s-process), that are of primary importance for the formation of heavy nuclides through a chain based on nuclear captures and successive $\beta$ decays. As it is shown in table 1 , the reactions cited above will be studied with the approved LUNA-MV accelerator.

\section{Big Bang Nucleosynthesis}

Big Bang Nucleosynthesis (BBN) theory describes the formation of light nuclides in the first minutes of cosmic time. Their abundance only depends on particle physics, baryon density and nuclear processes of BBN chain. Indeed, the comparison of computed and observed abundances of light elements is a powerful tool to constraint particle physics, astrophysics and cosmology, provided that the knowledge of the cross sections of the relevant nuclear reactions is accurate enough. In this framework the LUNA measurements represent a sizable step forward for the BBN theory, providing a direct experimental footing to calculate the primordial abundances of $\mathrm{D},{ }^{3} \mathrm{He},{ }^{6} \mathrm{Li}$ and ${ }^{7} \mathrm{Li}$ (see table 1). As an example, the study of the $d(p, \gamma)^{3} \mathrm{He}$ reaction made possible to reduce of a factor three the uncertainty of the computed deuterium abundance $(D / H)_{b b n}$ [34]. In turn, the comparison of $(D / H)_{b b n}$ with the abundance obtained from direct observations $(D / H)_{o b s}$ allows to measure the universal baryon density $\Omega_{b}$, to constrain the number of neutrino species $N_{\text {eff }}$ and 


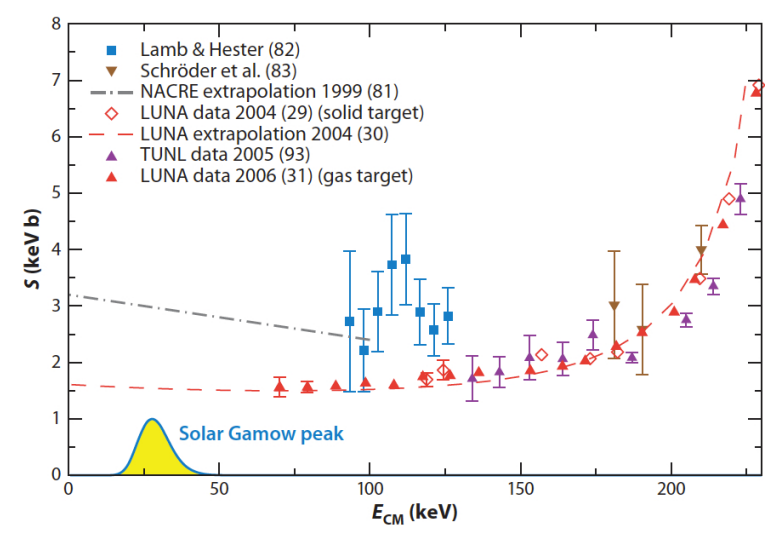

Figure 6: Astrophysical S-factor for the ${ }^{14} N(p, \gamma){ }^{15} O$. The LUNA measurements are shown together with previous measurements (see [18] and references therein).

the lepton degeneracy $[32,36,37]$. A renewed measurement of the $d(p, \gamma)^{3} \mathrm{He}$ reaction in the BBN energy region is presently mandatory to improve the $(D / H)_{b b n}$ accuracy. In fact, $D / H_{o b s}$ has been recently determined at the percent level with new deuterium observations and analysis in high redshift QSO absorbers [36] and $\Omega_{b}$ has been inferred from the PLANCK data [35], providing an independent estimate of this parameter. The $d(p, \gamma)^{3} \mathrm{He}$ measurement is presently in progress by means of the $400 \mathrm{kV}$ accederator. The goal is to reach a $3 \%$ accuracy in the astrophysical factor measurement, for $E_{c m} \leqslant 266 \mathrm{keV}[32,33]$. Finally, the LUNA-MV project also foresees a renewed study of the ${ }^{3} \mathrm{He}\left({ }^{4} \mathrm{He}, \gamma\right){ }^{7} \mathrm{Be}$ reaction and it is well tailored to study of the ${ }^{2} \mathrm{H}(\alpha, \gamma){ }^{6} \mathrm{Li}$ process in the whole BBN energy range. Those measurements are of crucial importance to face the so called "Lithium problem", i.e. the discrepancies between observed and computed abundances of these fragile isotopes.

\section{Conclusion}

Reactions of primary importance in astrophysics has been measured by the LUNA collaboration at the lowest energies ever reached, taking advantage of the ultra-low background achievable at the underground Gran Sasso laboratory. The future of LUNA is a natural evolution of the present activity. In this concern, the LUNA-MV accelerator should substantially improve our understanding of advanced burning in stars, because of the possibility of measuring for the first time the cross section of key reactions at energies close to the Gamow peak of typical stellar environments.

\section{References}

[1] C. Rolfs and W.S. Rodney: Cauldrons in the cosmos, University of Chicago Press (1988).

[2] C. Iliadis, Nuclear Physics of Stars (Weinheim:Wiley-VCH) (2007).

[3] U. Greife et al, NIM A 350 (1994). Volume 350, 327Đ337, (1994). 
[4] R. Bonetti et al, Phys. Rev. Lett. 82, 5205 (1999).

[5] C. Casella et al, Nuclear Physics A 706 203Đ216. (2002).

[6] P. Prati et al, Z. Phys. A 350, 171-176 (1994).

[7] H. Costantini et al, Phys. Lett. B $48243 Đ 49$ (2000).

[8] M. Aliotta et al, Nucl. Phys. A $690790 Đ 800$ (2001).

[9] A. Formicola et al, Nucl. Instr. and Meth. A 507609 (2003).

[10] J.N. Bahcall, M.H. Pinsonneault, Rev. Mod. Phys. 64, 885 (1992).

[11] W.A. Fowler, Nature 238, 24 (1972).

[12] D. Bemmerer et al, Phys. Rev. Lett. 97, 122502 (2006).

[13] F. Confortola et al, Phys. Rev. C 75, 065803 (2007).

[14] A. Formicola et al, Phys. Lett. B 591, 61 (2004).

[15] G. Imbriani AA 420 625Đ629 (2004).

[16] G. Imbriani et al, Eur. Phys. J. A, 25 455-466 (2005).

[17] M. Marta et al, Phys. Rev. C 78, 022802 (2008).

[18] A. Lemut et al, Phys. Lett. B 634483 (2006).

[19] B.Limata et al, Phys. Rev. C 82, 015801 (2010).

[20] F. Strieder et al, Phys. Lett. B 7070 60-65 (2012).

[21] O.Straniero et al, The Astrophysical Journal, 763:100 (10pp) (2013).

[22] D.Bemmerer et al, J. Phys. G: Nucl. Part. Phys. 36045202 (2009).

[23] A.Caciolli et al, AA 533, A66 (2011).

[24] D.A. Scott et al, Phys. Rev. Lett. 109, 202501 (2012).

[25] A. Di Leva et al, Phys. Rev. C 89, 015803 (2014).

[26] "LUNA proposal 2008-2012", http://luna.Ings.infn.it/Documents/proposal2008.pdf.

[27] M. Anders et al, Eur. Phys. J. A 4928 (2013).

[28] M. Anders et al, PRL 113, 042501 (2014).

[29] "LUNA proposal 20015-2018", submitted to the LNGS scientific committee.

[30] The LUNA Collaboration, LUNA-MV Letter of Intent- LoI 42/07

[31] The LUNA Collaboration, Addendum to the LUNA-MV Letter of Intent- LoI 42/07.

[32] E. Di Valentino, C. Gustavino et al., Phys. Rev. D 90, 023543 (2014).

[33] C. Gustavino, "BBN, Neutrinos and Nuclear Astrophysics" PoS(Bormio 2014)050.

[34] P.D. Serpico et al, J. Cosmol. Astropart. Phys. 2004, 010 (2004).

[35] PLANCK collaboration: arXiv:1502.01589v2 [astro-ph.CO] 6 Feb 2015.

[36] R. Cooke et al, ApJ. 781, 31, (2014).

[37] K.M. Nollett and G.P. Holder: arXiv:1112.2683v1 [astro-ph.CO] 12 Dec 2011. 